\title{
Retraction Note to: Nucleolin Promotes TGF- $\beta$ Signaling Initiation via TGF- $\beta$ Receptor I in Glioblastoma
}

\author{
Shunzeng Lv ${ }^{1,2}$ • Jie Zhang ${ }^{1,3} \cdot$ Mingzhi Han ${ }^{2}$ - Weiping Wang ${ }^{4} \cdot$ Ya Zhang $^{2}$ • \\ Dongxiao Zhuang ${ }^{5} \cdot$ Ranran $_{S^{2}}{ }^{2} \cdot$ Ruixiang Bian ${ }^{2} \cdot$ Chengjun Yao ${ }^{5}$
}

Published online: 15 June 2017

(C) Springer Science+Business Media, LLC 2017

\section{Retraction Note to: J Mol Neurosci (2015) 55:1-6 DOI 10.1007/s12031-014-0292-9}

This article has been retracted at the request of Dr. Chengjun Yao, Dr. Dongxiao Zhuang, The Editor-in-Chief and the Publisher per the Committee on Publication Ethics guidelines for the following reasons:

- There is strong reason to believe that the peer review process was compromised.
- The article shows evidence of irregularities in authorship during the submission process.

- Dr. Chengjun Yao has confirmed that he was not involved in the submission process and does not support its publication.

- Dr. Dongxiao Zhuang has confirmed that he was not involved in the research nor in the writing of this article.

- The National Natural Science Foundation of China mentioned in this paper was not involved in the research reported in this article

The online version of the original article can be found at http://dx.doi:10. 1007/s12031-014-0292-9

Chengjun Yao

cjyhuashan@163.com

1 Peking Union Medical College Hospital, Chinese Academy of Medical Sciences \& Peking Union Medical College, Beijing, China

2 Shandong University School of Medicine, Jinan, Shandong, China

3 The First Clinical College of Nanjing Medical University, Nanjing, China

4 Department of Radiotherapy, Peking Union Medical College Hospital, Chinese Academy of Medical Sciences \& Peking Union Medical College, Beijing, China

5 Glioma Surgery Division, Neurological Surgery Department, Huashan Hospital, Shanghai Medical College of Fudan University, Shanghai 200040, China 